\title{
Adsorption and photo catalytic degradation of yellow Auramine-O dye using carbon nano particles
}

\author{
V. Latha \\ Department of Chemistry, Sri S. Ramasamy Naidu Memorial College, Sattur, India \\ latha@srnmcollege.ac.in
}

PACS 61.46.+W

DOI 10.17586/2220-8054-2021-12-3-363-367

In this study, Carbon nano particles (C NPs) from neem leaves were synthesized for adsorption and photo catalytic degradation of Auramine-O (Au-O) dye used in paper industries. The synthesized C NPs were characterized by powder X-ray diffraction and scanning electron microscopy. Dye adsorption and photo catalytic properties of $\mathrm{C} \mathrm{NPs}$ were examined by studying the decolorization of dye Au-O at 5 minutes time interval using a UV-Visible spectrophotometer. The results show that C NPs acts as a better adsorbent even with shorter time and lower concentration than as photocatalysts.

Keywords: Dye degradation, carbon nano particle, adsorption, photo catalytic activity, degradation of Auramine-O dye, X-ray diffraction, photo catalysts.

Received: 15 March 2021

Revised: 16 April 2021

\section{Introduction}

Synthetic dyes have been widely used in many technological processes such as paints and textiles, as an additive in plastic, paper, leather, cosmetics and rubber industries, and so on [1]. The paper industry is one of the oldest industries in which the extensive use of various dyes has been implemented for ages. There are many variations in the kind of papers produced in paper industry such as writing and printing paper, tissues used as toilet paper, facial tissues or used as paper napkins or towel, copier paper, cover papers, corrugated papers used for packing, handmade paper, lamination paper and the list is endless. For each kind of paper, a different kind of dye is required. The dying process in poly board paper industry involves many dyes such as Acid orange, Brown dye, Red dye, Paper yellow, Methylene Blue, Victoria Pure Blue, Malachite Green Crystals, Rhodamine 6, Methyl Violet, Rhodamine B, Auramine O. Out of these, Auramine-O (Au-O) is a basic yellow dye containing amino and imino groups (Fig. 1). The effluents containing $\mathrm{Au}-\mathrm{O}$ cause significant health problems. Au-O may bind to hemoglobin, inhibiting the normal uptake of oxygen, leading to methemoglobinemia, cyanosis and breathing difficulties. There is evidence that it may produce eye irritation and eye damage 24 hours or more after instillation. There may be damage to the cornea. Unless treatment is prompt and adequate there may be permanent loss of vision. Inhalation leads to unconscious. Continuous exposure to $\mathrm{Au}-\mathrm{O}$ causes vomiting and yellowish discoloration of skin [2].<smiles>CN(C)c1ccc(C(=N)c2ccc(N(C)C)cc2)cc1</smiles>

FIG. 1. Structure of Auramine-O

Even at low concentration, the colors of these dyes can be easily observed by water and make it highly injurious effects for an environment and human health. Therefore, significant attention has been drawn to develop a beneficial technology for the removal of these organic pollutants from aqueous solutions. These harmful organic pollutants can be removed by various methods such as precipitation, adsorption, photocatalytic degradation, ion exchange, reverse osmosis, solvent extraction and chlorination. Adsorption of dyes by activated carbon and its composites is one of the simplest and most economical methods for removing dyes from wastewater due to its high adsorption capacity, 
high specific surface area, and low selectivity [3-15]. Previous research revealed that photocatalyst had shown a good performance in degrading the persistent organic pollutants with the aid of light absorption in the water [16].

However, the performance does depend not only on the composition, but also on its microstructure, size, and morphology. Smaller nanoparticles could form on active sites of support materials through oxide-support interaction. This could increase the overall surface area of the photocatalyst, and the support materials can act as a sink of electrons. In recent trends, nanomaterials are used as a photocatalyst to degrade organic pollutants. Nanoscale materials have high surface area to volume ratio, making them ideal for use in composite materials, drug delivery, catalysis, sensors, data storage and energy storage. The finite size of material entities as compared to the molecular scale makes nanostructured materials harder, less brittle and mechanically strong. Basically, in nanotechnology, we study materials at nanoscale $\left(1 \cdot 10^{-9} \mathrm{~m}\right)$, materials at bulk scale show different properties and at nanoscale different extraordinary properties, at nanoscale with size their surface to volume ratio increase. This surface to volume ratio property, especially as a catalyst, increases the efficiency by a hundred percent as compared to bulk materials.

Carbon based nanoparticles have application as photocatalysts but there are some limitations, due to which, these were not use commercially. So the present work focussed on the synthesis of carbon nanoparticles from neem leaves and its adsorption and photocatalytic action against $\mathrm{Au}-\mathrm{O}$ dye.

The neem (Azadirachta indica A. Juss) is a tropical evergreen tree traditionally well known for its medicinal value. Apart from Indian subcontinent, neem is widely used in African countries as therapeutics, preservatives and insecticides. Neem leaves, natural source of flavonoids, polyphenols, isoprenoids, sulfurous and polysaccharides, play important role in free radical scavenging. Neem leaves are used for treating leprosy, eye disorders, bloody nose, intestinal worms, stomach upset, skin ulcers, fever, diabetes, gum disease, and liver problems.

$\mathrm{As} \mathrm{Au}-\mathrm{O}$ dye causes skin coloring, vomiting, unconsciousness, the carbon nanoparticles synthesized from neem leaves may be the best adsorbent.

\section{Materials and methods}

\subsection{Synthesis of carbon nanoparticles}

Carbon nanoparticles were synthesized from neem leaves. The fresh neem leaves were collected and then washed with distilled water. Then the washed leaves were soaked in dilute hydrochloric acid for 3-4 hours. After 4 hours the color of the leaves turned black, at which point they were collected and dried at $120^{\circ} \mathrm{C}$ for $3-5$ hours in a hot air oven. The dried mass was collected and stored for application.

\subsection{Adsorption}

The adsorption performance of carbon nanoparticle was evaluated through the decreased UV absorbency of Au-O solution. The chemical structure of Au-O is shown in Fig. 1. The concentration of Au-O was 5 PPM. $5 \mathrm{~mL}$ of the dye solution is sonicated with $20 \mathrm{mg}$ of carbon nanoparticle to get better adsorption activity. The absorbency of the Au-O solution at a wavelength of $435 \mathrm{~nm}$ was measured every $10 \mathrm{~min}$ by a UV-vis spectrophotometer for 50 minutes.

\subsection{Photocatalytic with UV and Visible Sources}

Photocatalytic degradation process was also carried out under UV light irradiation. Four fluorescent lamps were used for irradiation of UV light source. $50 \mathrm{~mL} \mathrm{Au-O}$ dye solution with 5 PPM concentration was sonicated continuously with $20 \mathrm{mg}$ of Carbon nanoparticles in a $100 \mathrm{~mL}$ cylindrical Pyrex vessel reactor. The reaction was initiated by switching on the light source. $5 \mathrm{ml}$ of dye solution was taken at the equal intervals of 5 minutes from 0 upto 30 minutes. To examine the degradation of $\mathrm{Au}-\mathrm{O}$, the collected solutions were filtered and the UV-Vis absorption spectra were recorded by UV-Vis spectrometer.

\subsection{Characterization}

Scanning Electron Microscope EVO18 (CARL ZEISS) was used to investigate the surface morphology of carbon nanoparticles. Scanning electron microscopy (SEM) is a widely used method for the high-resolution imaging of surfaces that can also be employed to characterize nanoscale materials. SEM uses electrons for imaging, much as a light microscope uses visible light. The sample were placed in an evacuated chamber and scanned in a controlled pattern by an electron beam. Interaction of the electron beam with specimen produces the SEM images of Carbon NPs.

X-ray diffractometer D8 Advance ECO (Bruker) was used to investigate the grain size. X-ray diffraction (XRD) is one of the most extensively used techniques for the characterization of NPs. Typically, XRD provides information regarding the crystalline structure, nature of the phase, lattice parameters and crystalline grain size. The grain size is 


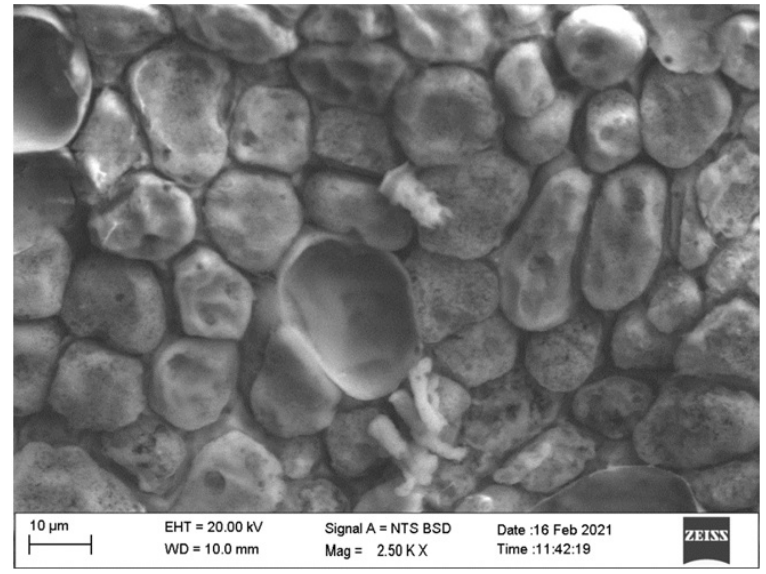

FIG. 2. SEM images of C NPs

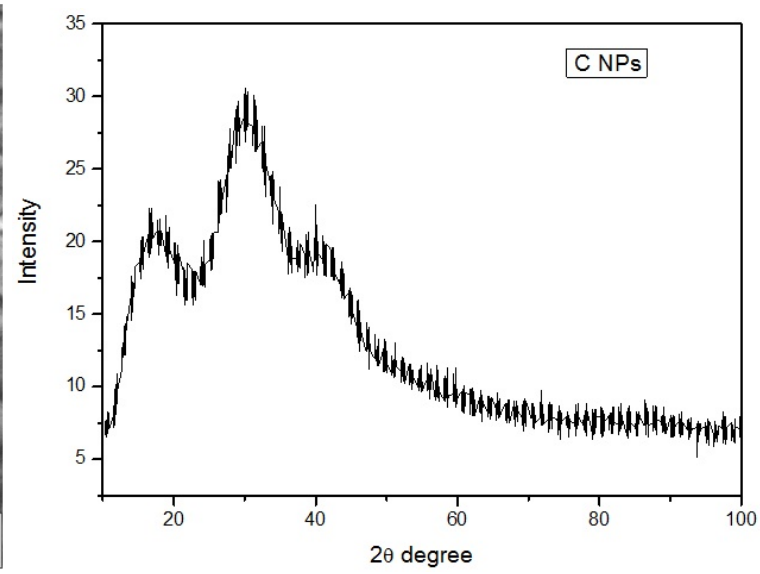

FIG. 3. XRD Pattern of Carbon NPs

estimated by using the Scherrer equation using the broadening of the most intense peak of an XRD measurement for a specific sample.

\section{Result and Discussion}

\subsection{Scanning electron microscopic analysis}

Morphology of synthesized carbon nanoparticles was characterised by SEM analysis. The SEM images were developed by the interaction of electron beam with carbon nano particles (Fig. 2). The Carbon NPs formed are well dispersed and evenly distributed in all direction. It shows that the Carbon NPs have smooth and uniform surface. It also confirms that there is no contamination with carbon nano particles.

\subsection{X-ray diffraction analysis}

The XRD pattern of the synthesized $\mathrm{C}$ and $\mathrm{C}-\mathrm{CaO}$ NPs are shown in Fig. 3. It shows that the XRD pattern of the synthesized $C$ NPs observed with $2 \Theta$ values $16^{\circ} 58^{\prime}, 18^{\circ} 93^{\prime}, 30^{\circ} 15^{\prime}, 39^{\circ} 96^{\prime}$ they are indexed to the $221,100,111$, 220 crystal planes. The average size of the synthesized C NPs was calculated using Scherrer's equation:

$$
D=\frac{k \lambda}{\beta \cos \Theta},
$$

where, $D$ - particle in size, $k$ - Scherrer's coefficient, $\lambda$ - wavelength of X-ray source (1.5406 nm), $\beta$ - full width half maximum (FWHM) and $\Theta$ - diffraction angle.

The average size of carbon nano particle is found to be $6.78 \mathrm{~nm}$. It shows that the synthesized Carbon NPs are spherical.

\subsection{Adsorption of dye molecule by Carbon NPs}

Adsorption of $\mathrm{Au}-\mathrm{O}$ dye molecule by Carbon NPs was examined. The adsorption peaks of the synthesized C NPs are depicted in Fig. 4. From the spectra, it is perceived that the intensity of the characteristic peak of Au-O observed at $435 \mathrm{~nm}$ decreases with respect to the time for the synthesized C NPs. This reduction in the concentration up to $55 \%$ of the initial concentration is due to the adsorption of Au-O dye by adsorption of Carbon nano particle. After 50 minutes, the concentration is reduced to $48 \%$ only.

\subsection{Photocatalytic degradation of dye molecule by Carbon NPs}

The photocatalytic performance of the synthesized C NPs is examined by the degradation of $\mathrm{Au}-\mathrm{O}$ dye molecules under UV-light irradiation. The photocatalytic degradation peaks of C NPs are depicted in Fig. 5. From the spectra, the intensity of the characteristic peak of Au-O observed at $435 \mathrm{~nm}$ decreases with respect to the time for the synthesized C NPs. But the intensity is not that much decreased as in the case of adsorption. This study implies that C NPs are acting as better adsorbents than as photocatalysts. 


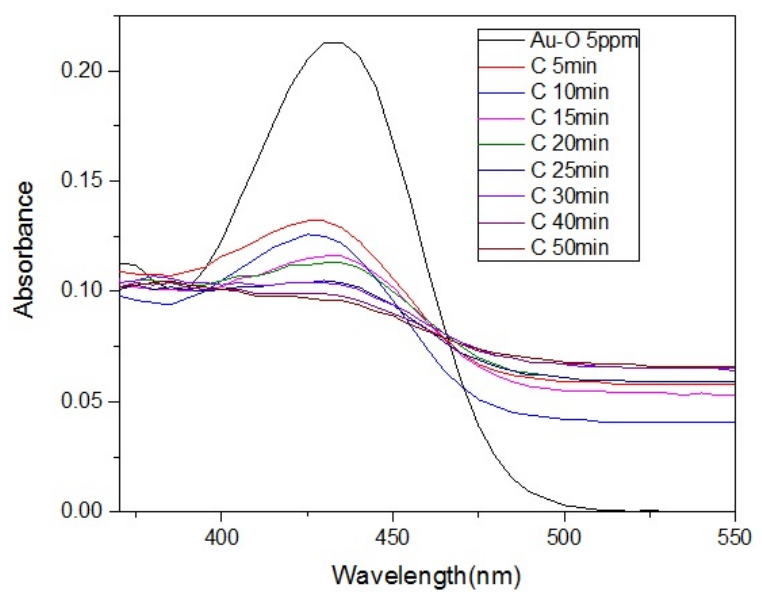

FIG. 4. UV - Visible spectra of $\mathrm{Au}-\mathrm{O}$ with $\mathrm{C}$ NPs at time interval of 5 minutes from 0 to 50 minutes by adsorption studies

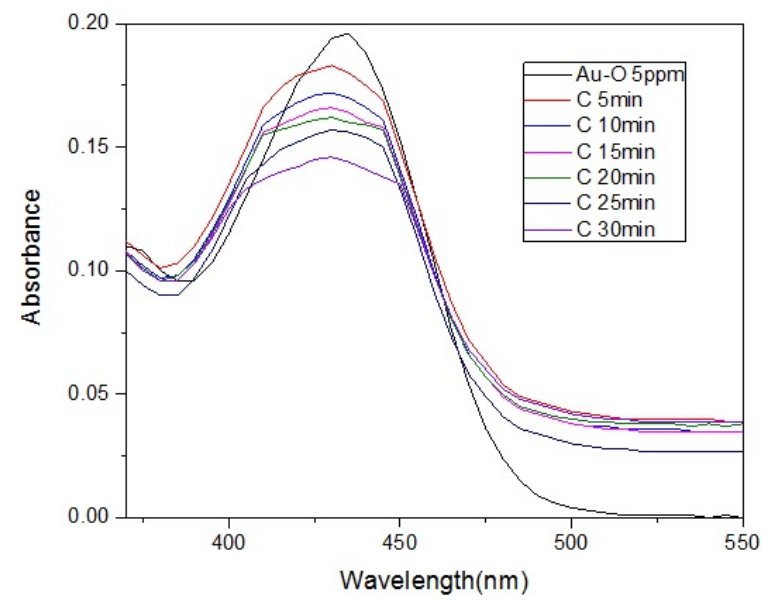

FIG. 5. UV - Visible spectra of $\mathrm{Au}-\mathrm{O}$ with $\mathrm{C}$ NPs at time interval of 5 minutes from 0 to 30 minutes

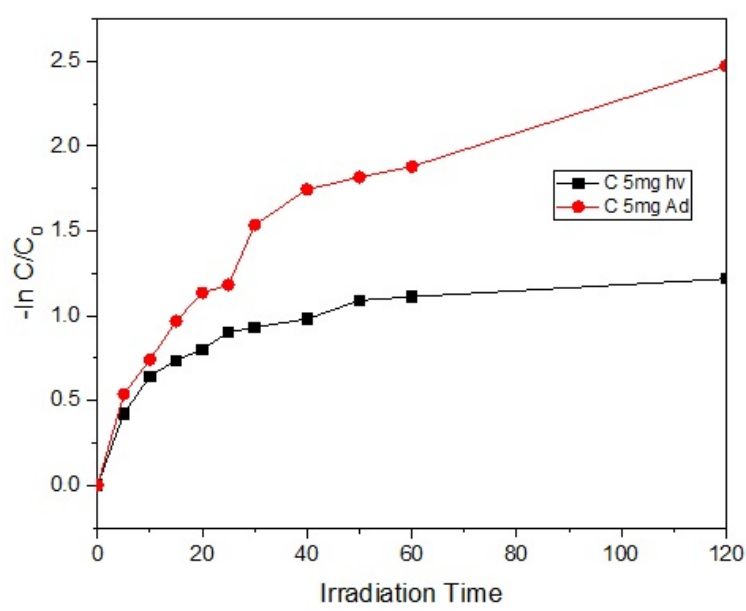

FIG. 6. Time course of Au-O adsorption on C NPs for both adsorption and photocatalytic degradation 


\subsection{Mechanism for Au-O degradation by Carbon NPs}

Fig. 6 depicts the time course of Au-O degradation on $\mathrm{C}$ NPs by adsorption and photocatalytic degradation process. Compared to the photocatalytic process, the adsorption process has significantly degraded the $\mathrm{Au}-\mathrm{O}$ due to C NPs nano size, highly porous structures, in terms of area, pore volume, and pore distribution. The adsorption equilibrium was achieved after $40 \mathrm{~min}$. The graph matches with the physical adsorption curve of Langmuir. But in the photocatalysis process, the low performance of degradation compared to the adsorption process may be due to the rise in temperature by passing the light radiation. This may cause desorption process. Thus, the C NPs act as better adsorbent than photocatalytic degrader.

\section{Conclusion}

In summary, the carbon nanoparticles were successfully synthesised from neem leaves with smooth and uniform morphology with $6.28 \mathrm{~nm}$ grain size. These carbon nanoparticles act as better adsorbents than as photocatalysts. This leads to further study to synthesize composite material with this carbon nano particle to increase their photocatalytic activity.

\section{Acknowledgements}

This work was supported by the 2019 TNSCST-S\&T Project Grant (Ref.No.TNSCST/STP/AR/2018-2019/9298 dt.04.11.2019) from TamilNadu State Council for Science and Technology, Chennai. The author acknowledges TNSCST, Chennai for extending financial support and Sri. SRNM College, Sattur, for providing necessary infrastructures to carry out this work.

\section{References}

[1] K. Hunger Industrial Dyes: Chemistry, Properties, Applications. John Wiley \& Sons, 2007.

[2] S. Kaja Mohideen, K. Senthil Kumar, D. Pavan Kumar. Cow dung powder poisoning. Indian Journal of Critical Care Medicine, 2015, 15, P. 64-66.

[3] S.K. Giri, N.N. Das, and G.C. Pradhan. Synthesis and characterization of magnetite nanoparticles using waste iron ore tailings for adsorptive removal of dyes from aqueous solution. Colloid Surface Physicochemal Engineering Aspect, 2011, 389, P. $43-49$.

[4] P. Hu, Y. Zhang, K. Tong et al. Removal of organic pollutants from red water by magnetic-activated coke. Desalination and Water Treatment, 2014, 54, P. 2710-2722.

[5] T. Jiang, Y.-d. Liang, Y.-j. He, and Q. Wang. Activated carbon/ $\mathrm{NiFe}_{2} \mathrm{O}_{4}$ magnetic composite: a magnetic adsorbent for the adsorption of methyl orange. Journal of Environmental Chemical Engineering, 2015, 3, P. 1740-1751.

[6] T. Madrakian, A. Afkhami, M. Ahmadi, and H. Bagheri. Removal of some cationic dyes from aqueous solutions using magnetic-modified multi-walled carbon nanotubes. Journal of Hazardous Materials, 2011, 196, P. 109-114.

[7] H.R. Rajabi, H. Arjmand, S.J. Hoseini, and H. Nasrabadi. Surface modified magnetic nanoparticles as efficient and green sorbents: synthesis, characterization, and application for the removal of anionic dye. Journal of Magnetism and Magnetic Materials, 2015,394, P. 7-13.

[8] N. Yang, S. Zhu, D. Zhang, and S. Xu. Synthesis and properties of magnetic $\mathrm{Fe}_{3} \mathrm{O}_{4}$-activated carbon nanocomposite particles for dye removal. Materials Letters, 2008, 62, P. 645-647.

[9] B. Zargar, H. Parham, and A. Hatamie. Fast removal and recovery of amaranth by modified iron oxide magnetic nanoparticles. Chemosphere, 2009, 76, P. 554-557.

[10] A. Latif, S. Noor, Q.M. Sharif, and M. Najeebullah. Different techniques recently used for the treatment of textile dyeing effluents: a review. Journal of the Chemical Society of Pakistan, 2010, 32(1), P. 115-124.

[11] P. Pengthamkeerati, T. Satapanajaru, N. Chatsatapattayakul, P. Chairattanamanokorn, and N. Sananwai. Alkaline treatment of biomass fly ash for reactive dye removal from aqueous solution. Desalination, 2010, 261(1-2), P. 34-40.

[12] Z. Zhang and J. Kong. Novel magnetic $\mathrm{Fe}_{3} \mathrm{O}_{4} @ \mathrm{C}$ nanoparticles as adsorbents for removal of organic dyes from aqueous solution. Journal of Hazardous Materials, 2011, 193, P. 325-329.

[13] B. Zargar, H. Parham, and M. Rezazade. Fast removal and recovery of methylene blue by activated carbon modified with magnetic iron oxide nanoparticles. Journal of the Chinese Chemical Society, 2011, 58, P. 694-699.

[14] F. Liu, S. Chung, G. Oh, T.S. Seo. Three-dimensional graphene oxide nanostructure for fast and efficient water-soluble dye removal. ACS Applied Materials \& Interfaces, 2012, 4, P. 922-927.

[15] T. Madrakian, A. Afkhami, H. Mahmood-Kashani, and M. Ahmadi. Adsorption of some cationic and anionic dyes on magnetite nanoparticlesmodified activated carbon from aqueous solutions: equilibrium and kinetics study. Journal of the Iranian Chemical Society, 2013, 10, P. 481489.

[16] Pang Y.L., Lim S., Ong H.C., Chong W.T. Synthesis, characteristics and sonocatalytic activities of calcined $\gamma-\mathrm{Fe}_{2} \mathrm{O}_{3}$ and TiO ${ }_{2}$ nanotubes $\gamma-\mathrm{Fe}_{2} \mathrm{O}_{3}$ magnetic catalysts in the degradation of Orange G. Ultrason Sonochem, 2016, 29, P. 317-327. 